\title{
Deep Learning with Minimal Training Data: TurkuNLP Entry in the BioNLP Shared Task 2016
}

\author{
Farrokh Mehryary $^{1,3}$, Jari Björne ${ }^{2,3}$, Sampo Pyysalo ${ }^{4}$, Tapio Salakoski ${ }^{2,3}$ and Filip Ginter ${ }^{2,3}$ \\ ${ }^{1}$ University of Turku Graduate School (UTUGS) \\ ${ }^{2}$ Turku Centre for Computer Science (TUCS) \\ ${ }^{3}$ Department of Information Technology, University of Turku \\ Faculty of Mathematics and Natural Sciences, FI-20014, Turku, Finland \\ ${ }^{4}$ Language Technology Lab, DTAL, University of Cambridge \\ firstname.lastname@utu.fi, sampo@pyysalo.net
}

\begin{abstract}
We present the TurkuNLP entry to the BioNLP Shared Task 2016 Bacteria Biotopes event extraction (BB3-event) subtask. We propose a deep learningbased approach to event extraction using a combination of several Long Short-Term Memory (LSTM) networks over syntactic dependency graphs. Features for the proposed neural network are generated based on the shortest path connecting the two candidate entities in the dependency graph. We further detail how this network can be efficiently trained to have good generalization performance even when only a very limited number of training examples are available and part-of-speech (POS) and dependency type feature representations must be learned from scratch. Our method ranked second among the entries to the shared task, achieving an F-score of $52.1 \%$ with $62.3 \%$ precision and $44.8 \%$ recall.
\end{abstract}

\section{Introduction}

The BioNLP Shared Task 2016 was the fourth in the series to focus on event extraction, an information extraction task targeting structured associations of biomedical entities (Kim et al., 2009; Ananiadou et al., 2010). The 2016 task was also the third to include a Bacteria Biotopes (BB) subtask focusing on microorganisms and their habitats (Bossy et al., 2011). Here, we present the TurkuNLP entry to the BioNLP Shared Task 2016 Bacteria Biotope event extraction (BB3-event) subtask. Our approach builds on proven tools and ideas from previous tasks and is novel in its application of deep learning methods to biomedical event extraction.
The BB task was first organized in 2011, then consisting of named entity recognition (NER) targeting mentions of bacteria and locations, followed by the detection of two types of relations involving these entities (Bossy et al., 2011). Three teams participated in this task, with the best Fscore of $45 \%$ achieved by the INRA Bibliome group with the Alvis system, which used dictionary mapping, ontology inference and semantic analysis for NER, and co-occurrence-based rules for detecting relations between the entities (Ratkovic et al., 2011). The 2013 BB task defined three subtasks (Nédellec et al., 2013), the first one concerning NER, targeting bacteria habitat entities and their normalization, and the other two subtasks involving relation extraction, the task targeted also by the system presented here. Similarly to the current BB3-event subtask, the 2013 subtask 2 concerned only relation extraction, and subtask 3 extended this with NER. Four teams participated in these tasks, with the UTurku TEES system achieving the first places with F-scores of $42 \%$ and $14 \%$ (Björne and Salakoski, 2013).

We next present the 2016 BB3-event subtask and its data and then proceed to detail our method, its results and analysis. We conclude with a discussion of considered alternative approaches and future work.

\section{Task and Data}

In this section, we briefly present the BB3-event task and the statistics of the data that has been used for method development and optimization, as well as for test set prediction.

Although the BioNLP Shared Task has introduced an event representation that can capture associations of arbitrary numbers of participants in complex, recursive relationships, the BB3-event task follows previous BB series subtasks in ex- 


\begin{tabular}{lccc} 
& Train & Devel & Test \\
\hline Total sentences & 527 & 319 & 508 \\
Sentences w/examples & 158 & 117 & 158 \\
Sentences w/o examples & 369 & 202 & 350 \\
Total examples & 524 & 506 & 534 \\
Positive examples & 251 & 177 & - \\
Negative examples & 273 & 329 & -
\end{tabular}

Table 1: BB3-event data statistics. (The relation annotations of the test set have not been released.)

clusively marking directed binary associations of exactly two entities. For the purposes of machine learning, we thus cast the BB3-event task as binary classification taking either a (BACTERIA, HABITAT) or a (BACTERIA, GEOGRAPHICAL) entity pair as input and predicting whether or not a Livesin relation holds between the BACTERIA and the location (HABITAT or GEOGRAPHICAL).

Our approach builds on the shortest dependency path between each pair of entities. However, while dependency parse graphs connect words to others in the same sentence, a number of annotated relations in the data involve entities appearing in different sentences, where no connecting path exists. Such cross-sentence associations are known to represent particular challenges for event extraction systems, which rarely attempt their extraction (Kim et al., 2011). In this work, we simply exclude cross-sentence examples from the data. This elimination procedure resulted in the removal of 106 annotated relations from the training set and 62 annotated relations from the development set.

The examples that we use for the training, optimization and development evaluation of our method are thus a subset of those in the original data. ${ }^{1}$ When discussing the training, development and test data, we refer to these filtered sets throughout this manuscript. The statistics of the task data after this elimination procedure are summarized in Table 1. Note that since there are various ways of converting the shared task annotations into examples for classification, the numbers we report here may differ from those reported by other participating teams.

\footnotetext{
${ }^{1}$ Official evaluation results on the test data are of course comparable to those of other systems: any cross-sentence relations in the test data count against our submission as false negatives.
}

\section{Method}

We next present our method in detail. Preprocessing is first discussed in Section 3.1. Section 3.2 then explains how the shortest dependency path is used, and the architecture of the proposed deep neural network is presented in Section 3.3. Section 3.4 defines the classification features and embeddings for this network. Finally, in Section 3.5 we discuss the training and regularization of the network.

\subsection{Preprocessing}

We use the TEES system, previously developed by members of the TurkuNLP group (Björne and Salakoski, 2013), to run a basic preprocessing pipeline of tokenization, POS tagging, and parsing, as well as to remove cross-sentence relations. Like our approach, TEES targets the extraction of associations between entities that occur in the same sentence. To support this functionality, it can detect and eliminate relations that cross sentence boundaries in its input. We use this feature of TEES as an initial preprocessing step to remove such relations from the data.

To obtain tokens, POS tags and parse graphs, TEES uses the BLLIP parser (Charniak and Johnson, 2005) with the biomedical domain model created by McClosky (2010). The phrase structure trees produced by the parser are further processed with the Stanford conversion tool (de Marneffe et al., 2006) to create dependency graphs. The Stanford system can produce several variants of the Stanford Dependencies (SD) representation. Here, we use the collapsed variant, which is designed to be useful for information extraction and language understanding tasks (de Marneffe and Manning, 2008).

\subsection{Shortest Dependency Path}

The syntactic structure connecting two entities $e_{1}$ and $e_{2}$ in various forms of syntactic analysis is known to contain most of the words relevant to characterizing the relationship $R\left(e_{1}, e_{2}\right)$, while excluding less relevant and uninformative words.

This observation has served as the basis for many successful relation extraction approaches in both general and biomedical domain NLP (Bunescu and Mooney, 2005; Airola et al., 2008; Nguyen et al., 2009; Chowdhury et al., 2011). The TEES system also heavily relies on the shortest dependency path for defining and ex- 
tracting features (Björne et al., 2012; Björne and Salakoski, 2013). Recently, this idea was applied in an LSTM-based relation extraction system by $\mathrm{Xu}$ et al. (2015). Since the dependency parse is directed (i.e. the path from $e_{1}$ to $e_{2}$ differs from that from $e_{2}$ to $e_{1}$ ), they separate the shortest dependency path into two sub-paths, each from an entity to the common ancestor of the two entities, generate features along the two sub-paths, and feed them into different LSTM networks, to process the information in a direction sensitive manner.

To avoid doubling the number of LSTM chains (and hence the number of weights), we convert the dependency parse to an undirected graph, find the shortest path between the two entities (BACTERIA and HABITAT/GEOGRAPHICAL), and always proceed from the BACTERIA entity to the HABITAT/GEOGRAPHICAL entity when generating features along the shortest path, regardless of the order of the entity mentions in the sentence. With this approach, there is a single LSTM chain (and set of LSTM weights) for every feature set, which is more effective when the number of training examples is limited.

There is a subtle and important point to be addressed here: as individual entity mentions can consist of several (potentially discontinuous) tokens, the method must be able to select which word (i.e. single token) serves as the starting/ending point for paths through the dependency graph. For example, in the following training set sentence, "biotic surfaces" is annotated as a HABITAT entity:

\section{"We concluded that $S$. marcescens MG1 utilizes different regulatory systems and adhesins in attachment to biotic and abiotic surfaces [...]"}

As this mention consists of two (discontinuous) tokens, it is necessary to decide whether the paths connecting this entity to BACTERIA mentions (e.g., "S. marcescens $M G 1$ ") should end at "biotic" or "surfaces". This problem has fortunately been addressed in detail in previous work, allowing us to adopt the proven solution proposed by Björne et al. (2012) and implemented in the TEES system, which selects the syntactic head, i.e. the root token of the dependency parse sub-tree covering the entity, for any given multi-token entity. Hence, in the example above, the token "surfaces" is selected and used for finding the shortest dependency paths.

\subsection{Neural Network Architecture}

While recurrent neural networks (RNNs) are inherently suitable for modeling sequential data, standard RNNs suffer from the vanishing or exploding gradients problem: if the network is deep, during the back-propagation phase the gradients may either decay exponentially, causing learning to become very slow or stop altogether (vanishing gradients); or become excessively large, causing the learning to diverge (exploding gradients) (Bengio et al., 1994). To avoid this issue, we make use of Long Short-Term Memory (LSTM) units, which were proposed to address this problem (Hochreiter and Schmidhuber, 1997).

We propose an architecture centered around three RNNs (chains of LSTM units): one representing words, the second POS tags, and the third dependency types (Figure 1). For a given example, the sequences of words, POS tags and dependency types on the shortest dependency path from the BACTERIA mention to the HABITAT/GEOGRAPHICAL mention are first mapped into vector sequences by three separate embedding lookup layers. These word, POS tag and dependency type vector sequences are then input into the three RNNs. The outputs of the last LSTM unit of each of the three chains are then concatenated and the resulting higher-dimensional vector input to a fully connected hidden layer. The hidden layer finally connects to a single-node binary classification layer.

Based on experiments on the development set, we have set the dimensionality of all LSTM units and the hidden layer to 128 . The sigmoid activation function is applied on the output of all LSTM units, the hidden layer and the output layer.

\subsection{Features and Embeddings}

We next present the different embeddings defining the primary features of our model. In addition to the embeddings, we use a binary feature which has the value 0 if the corresponding location is a GEOGRAPHICAL entity and 1 if it is a HABITAT entity. This input is directly concatenated with the LSTM outputs and fed into the hidden layer. We noticed this signal slightly improves classification performance, resulting in a less than 1 percentage point increase of the F-score.

\subsubsection{Word embeddings}

We initialize our word embeddings with vectors induced using six billion words of biomedical 


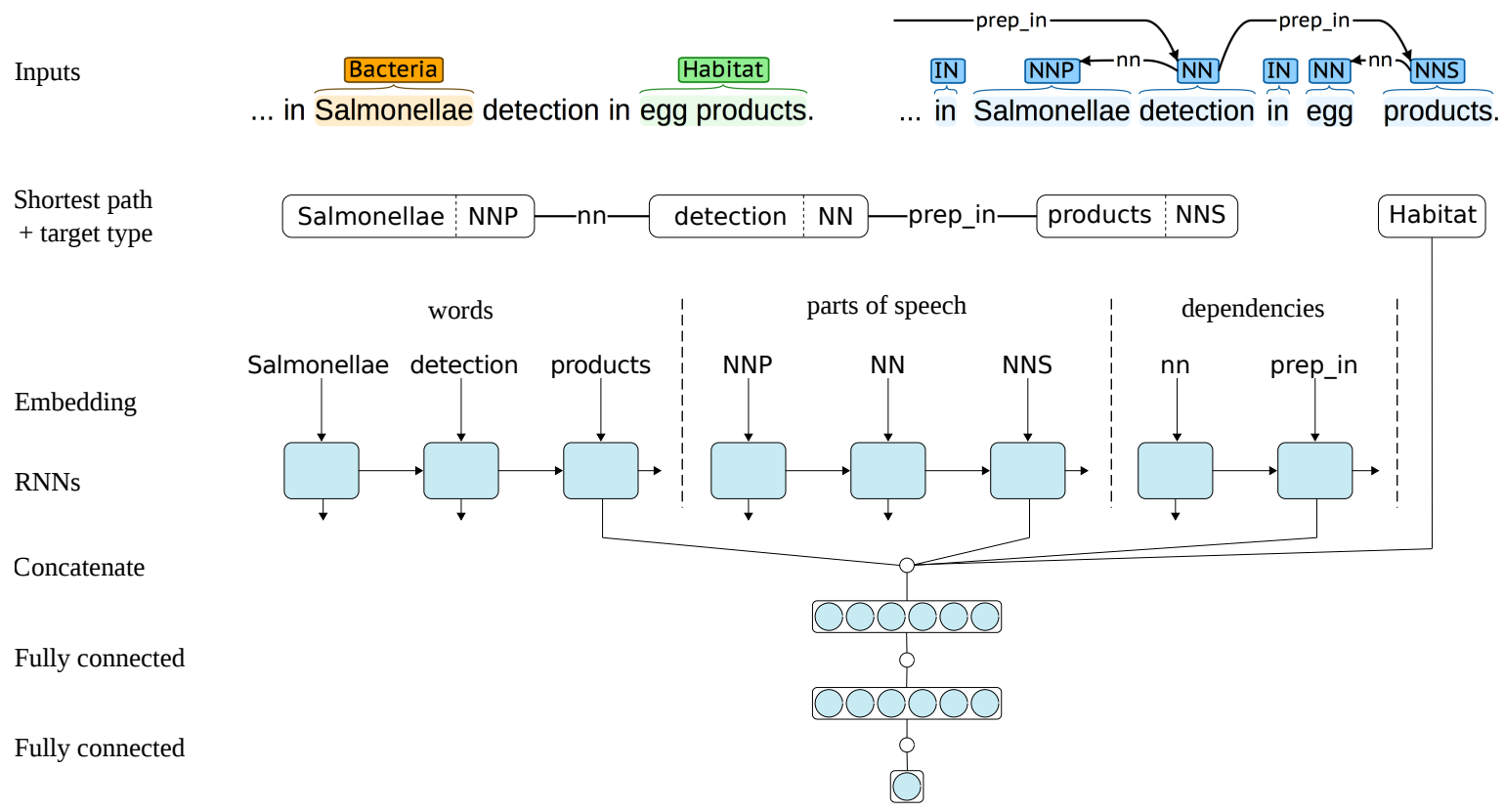

Figure 1: Proposed network architecture.

scientific text, namely the combined texts of all PubMed titles and abstracts and PubMed Central Open Access (PMC OA) full text articles available as of the end of September 2013. ${ }^{2}$ These 200-dimensional vectors were created by Pyysalo et al. (2013) using the word2 vec implementation of the skip-gram model (Mikolov et al., 2013).

To reduce the memory requirements of our method, we only use the vectors of the 100,000 most frequent words to construct the embedding matrix. Words not included in this vocabulary are by default mapped to a shared, randomly initialized unknown word vector. As an exception, out of vocabulary BACTERIA mentions are instead mapped to the vector of the word "bacteria". Based on development set experiments we estimate that this special-case processing improved the F-score by approximately $1 \%$ point.

\subsubsection{POS embeddings}

Our POS embedding matrix consists of a 100dimensional vector for each of the POS tags in the Penn Treebank scheme used by the applied tagger. We do not use pre-trained POS vectors but instead initialize the embeddings randomly at the beginning of the training phase.

\footnotetext{
${ }^{2}$ Available from http://bio.nlplab.org/
}

\subsubsection{Dependency type embeddings}

Typed dependencies - the edges of the parse graph - represent directed grammatical relations between the words of a sentence. The sequence of dependencies on the shortest path between two entities thus conveys highly valuable information about the nature of their relation.

We map each dependency type in the collapsed SD representation into a randomly initialized 350dimensional vector (size set experimentally). Note that in the applied SD variant, prepositions and conjunctions become part of collapsed dependency types (de Marneffe et al., 2006), as illustrated in Figure 2.

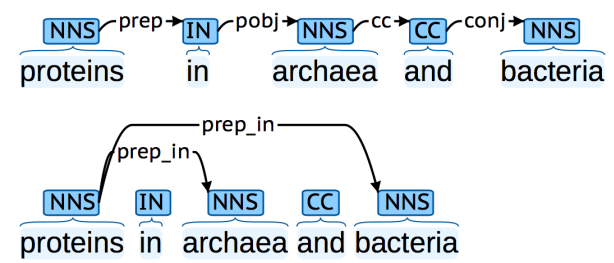

Figure 2: Basic (top) and collapsed (bottom) Stanford Dependency representations

As the collapsed dependencies thus incorporate preposition and conjunction words into the grammatical relations themselves, the set of dependency types is somewhat open-ended. To account for this, all preposition/conjunction dependency types not observed in the training and develop- 
ment sets are mapped to the vectors for the general preposition and conjunction types prep and conj, respectively.

\subsection{Training and Regularization}

We use binary cross-entropy as the objective function and the Adam optimization algorithm with the parameters suggested by Kingma and Ba (2014) for training the network. We found that this algorithm yields considerably better results than the conventional stochastic gradient descent in terms of classification performance.

During training, the randomly initialized POS and dependency type embeddings are trained and the pre-trained word embeddings fine-tuned by back-propagation using the supervised signal from the classification task at hand.

Determining how long to train a neural network model for is critically important for its generalization performance. If the network is under-trained, model parameters will not have converged to good values. Conversely, over-training leads to overfitting on the training set. A conventional solution is early stopping, where performance is evaluated on the development set after each set period of training (e.g. one pass through the training set, or epoch) to decide whether to continue or stop the training process. A simple rule is to continue while the performance on the development set is improving. By repeating this approach for 15 different runs with different initial random initializations of the model, we experimentally concluded that the optimal length of training is four epochs. Overfitting is a serious problem in deep neural networks with a large number of parameters. To reduce overfitting, we experimented with several regularization methods including the $l_{1}$ weight regularization penalty $(L A S S O)$ and the $l_{2}$ weight decay (ridge) penalty on the hidden layer weights. We also tried the dropout method (Srivastava et al., 2014) on the output of LSTM chains as well as on the output of the hidden layer, with a dropout rate of 0.5 . Out of the different combinations, we found the best results when applying dropout after the hidden layer. This is the only regularization method used in the final method.

\section{Results}

\subsection{Overcoming Variance}

At the beginning of training, the weights of the neural network are initialized randomly. As we are

\begin{tabular}{c|ccc} 
Run & Recall & Precision & F-score \\
\hline 12 & 76.3 & 60.3 & $\mathbf{6 7 . 3}$ \\
14 & 71.2 & 63.0 & 66.8 \\
13 & 75.7 & 59.3 & 66.5 \\
10 & 78.0 & 56.3 & 65.4 \\
3 & $\mathbf{8 0 . 8}$ & 54.0 & 64.7 \\
15 & 79.1 & 54.3 & 64.4 \\
1 & 66.1 & 62.2 & 64.1 \\
11 & 65.0 & 62.8 & 63.9 \\
2 & 67.8 & 59.4 & 63.3 \\
5 & 55.9 & 69.7 & 62.1 \\
7 & 57.6 & 66.7 & 61.8 \\
9 & 53.1 & 70.2 & 60.5 \\
8 & 50.9 & 74.4 & 60.4 \\
6 & 50.3 & 73.6 & 59.7 \\
4 & 46.9 & $\mathbf{7 8 . 3}$ & 58.7 \\
\hline $\bar{x}$ & 65.0 & 64.3 & 63.3 \\
$\sigma$ & 11.3 & 7.3 & 2.6
\end{tabular}

Table 2: Development set results for 15 repetitions with different initial random initializations with mean $(\bar{x})$ and standard deviation $(\sigma)$. Results are sorted by F-score.

only using pre-trained embeddings for words, this random initialization applies also to the POS and dependency type embeddings. Since the number of weights is high and the training set is very small (only 524 examples), the initial random state of the model can have a significant impact on the final model and its generalization performance. Limited numbers of training examples are known to represent significant challenges for leveraging the full power of deep neural networks, and we found this to be the case also in this task.

To study the influence of random effects on our model, we evaluate it with 15 different random initializations, training each model for four epochs on the training data and evaluating on the development set using the standard precision, recall and F-score metrics. Table 2 shows the obtained results. We find that the primary evaluation metric, the F-score, varies considerably, ranging from $58.7 \%$ to $67.3 \%$. This clearly illustrates the extent to which the random initialization can impact the performance of the model on unseen data. While the method is shown to obtain on average an Fscore of $63.3 \%$ on the development set, it must be kept in mind that given the standard deviation of 2.6, individual trained models may perform substantially better (or worse). It is also important to note that due to the small size of the development 


\begin{tabular}{c|ccc} 
Threshold $(t)$ & Recall & Precision & F-score \\
\hline 1 & $\mathbf{8 3 . 6}$ & 53.2 & 65.1 \\
2 & 79.7 & 54.0 & 64.4 \\
3 & 78.5 & 57.0 & 66.0 \\
4 & 78.0 & 59.0 & $\mathbf{6 7 . 2}$ \\
5 & 75.7 & 60.1 & 67.0 \\
6 & 70.6 & 60.7 & 65.3 \\
7 & 67.8 & 61.5 & 64.5 \\
8 & 65.5 & 62.0 & 63.7 \\
9 & 62.2 & 65.5 & 63.8 \\
10 & 58.2 & 66.5 & 62.1 \\
11 & 57.1 & 69.7 & 62.7 \\
12 & 52.5 & 70.5 & 60.2 \\
13 & 51.4 & 72.8 & 60.3 \\
14 & 48.6 & 74.8 & 58.9 \\
15 & 45.2 & $\mathbf{8 0 . 0}$ & 57.8
\end{tabular}

Table 3: Development set results for voting based on the predictions of the 15 different classifiers. Best results for each metric shown in bold.

set, individual models that achieved high performance in this experiment will not necessarily generalize well to unseen data.

To deal with these issues, we introduce a straightforward voting procedure that aggregates the prediction outputs of the 15 classifiers based on a given threshold value $t \in\{1, \ldots, 15\}$ :

1. For each example, predict outputs with the 15 models;

2. If at least $t$ outputs are positive, label the example positive, otherwise label it negative.

Clearly, the most conservative threshold is $t=15$, where a relation is voted to exist only if all the 15 classifiers have predicted it. Conversely, the least conservative threshold is $t=1$, where a relation is voted to hold if any classifier has predicted it.

The development set results for the voting algorithm with different threshold values are given in Table 3. As expected, the threshold $t=1$ produces the highest recall $(83.6 \%)$ with the lowest precision $(53.2 \%)$. With increasing values of $t$, precision increases while recall drops, and the highest precision $(80.0 \%)$ is achieved together the lowest recall of $(45.2 \%)$ with $t=15$. The best F-score is obtained with $t=4$, where an example is labeled positive if at least four classifiers have predicted it to be positive and negative otherwise. Figure 3 shows the precision-recall curve for these 15 threshold values.

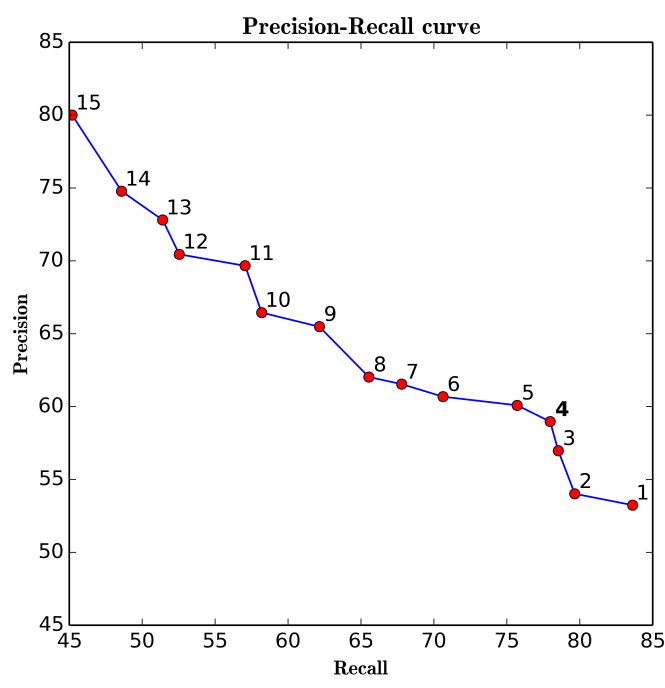

Figure 3: Precision-recall curve for different values of the threshold $t$ (shown as labels on the curve).

As is evident from these results, the voting algorithm can be used for different purposes. If the aim is to obtain the best overall performance, we can investigate which threshold produces the highest F-score (here $t=4$ ) and select that value when making predictions for unseen data (e.g., the test set). Alternatively, for applications that specifically require high recall or high precision, a different threshold value can be selected to optimize the desired metric.

To assess the performance of the method on the full, unfiltered development set that includes also cross-sentence relations, we selected the threshold value $t=4$ and submitted the aggregated prediction results to the official Shared Task evaluation server. The method achieved an F-score of $60.0 \%$ (60.9\% precision and $59.3 \%$ recall), $7.2 \%$ points below the result for our internal evaluation with filtered data (Table 3).

\subsection{Test Set Evaluation}

For evaluation of the test set, we applied the proposed model with the voting approach presented above): 15 neural network models with different random initializations were trained for 4 epochs on the combination of the training and the development sets. Each trained model was then used to produce one set of predictions for the test set. To obtain the final test set predictions, the outputs of the 15 classifiers were aggregated using the voting algorithm with a threshold $t=4$. 
Our method achieved an F-score of $52.1 \%$ with a recall of $44.8 \%$ and precision of $62.3 \%$, ranking second among the entries to the shared task. We again emphasize that our approach ignored all potential relations between entities belonging to different sentences, which may in part explain the comparatively low recall.

\subsection{Runtime Performance and Technical Details}

We implemented the model using the Python programming language (v2.7) with Keras, a modellevel deep learning library (Chollet, 2015). All network parameters not explicitly discussed above were left to their defaults in Keras. The Theano tensor manipulation library (Bastien et al., 2012) was used as the backend engine for Keras. Computations were run on a single server computer equipped with a GPU. ${ }^{3}$ All basic python processing, including e.g. file manipulation, the TEES pipeline and our voting algorithm, was run on a single CPU core, while all neural network related calculations (training, optimization, predictions) were run on the GPU, using the CUDA toolkit version 5.0.

The training process takes about 10 minutes, including model building and 4 epochs of training the network on the training set, but excluding preprocessing and the creation and loading of the input network. Prediction of the development set using a trained model with fully prepared inputs is very fast, taking only about 10 seconds. Finally, the voting algorithm executes in less than a minute for all 15 thresholds.

We note that even though the proposed approach involving 15 rounds of training, prediction and result aggregation might seem to be impractical for large-scale real-word applications (e.g., extracting bacteria-location relations from all PubMed abstracts), it is quite feasible in practice, as the time-consuming training process only needs to be done once, and prediction on unseen data is quite fast.

\subsection{Other Architectures}

In this section, we discuss alternative approaches that we considered and the reasons why they were rejected in favor of that described above.

\footnotetext{
${ }^{3}$ In detail: two 6-core Intel ${ }^{\circledR}$ Xeon ${ }^{\circledR}$ E5-2620 processors, 32 gigabytes of main memory, and one NVIDIA ${ }^{\circledR}$ TESLA $^{\mathrm{TM}}$ C2075 companion processor with 448 CUDA cores and 6 gigabytes of memory.
}

One popular and proven method for relation extraction is to use three groups of features, based on the observation that the words preceding the first entity, the words between the entities, and those after the second entity serve different roles in deciding whether or not the entities are related (Bunescu and Mooney, 2006). Given a sentence $S=w_{1}, \ldots, e_{1}, \ldots, w_{i}, \ldots, e_{2}, \ldots, w_{n}$ with entities $e_{1}$ and $e_{2}$, one can represent the sentence with three groups of words: $\{$ before $\} e_{1}\{$ middle $\} e_{2}\{$ after $\}$ ( $e_{1}$ and $e_{2}$ can also be included in the groups). The similarity of two examples represented in this way can be compared using e.g. sub-sequence kernels at word level (Bach and Badaskar, 2007). Bunescu and Mooney (2006) utilize three subkernels matching combinations of the before, middle and after sequences of words, with a combined kernel that is simply the sum of the subkernels. This kernel is then used with support vector machines for relation extraction. Besides the words, other features such as the corresponding POS tags and entity types can also be incorporated into such kernel functions to further improve the representation.

We adapted this idea to deep neural networks. We started with the simplest architecture, which contains 3 LSTM networks. Instead of generating features based on the shortest path, each LSTM receives inputs based on the sequence of the words seen in each of the before, middle, and after groups, where the word embeddings are the only features used for classification. Similar to the architecture discussed in Section 3.3, the outputs of the last LSTM units in each chain are concatenated, and the resulting higher-dimensional vector is then fed into a fully connected hidden layer and then to the output layer. This approach has a major advantage over the shortest dependency path, in particular for large-scale applications: parsing, the most time-consuming part in the relation extraction pipeline, is no longer required.

Unfortunately, our internal evaluation on the development set showed that this model failed to achieve results comparable to those of the shortest dependency path model, only reaching an F-score of about $57 \%$. Hence, we attempted to use more features by adding 3 or 6 additional LSTM chains to the model, for POS or/and dependency type embeddings. Even in these cases, the F-scores only varied in the range of $57 \%$ to about $63 \%$ (for different random initializations). We conclude 
that even though not requiring parsing is a benefit in these approaches, our experiments suggest that they are not capable of reaching performance comparable to methods that use the syntactic structure of sentences.

\section{Conclusions and Future work}

We have presented the entry of the TurkuNLP team to the Bacteria Biotope event extraction (BB3-event) sub-task of the BioNLP Shared Task 2016. Our method is based on a combination of LSTM networks over syntactic dependency graphs. The features for the network are derived from the POS tags, dependency types, and word forms occurring on the shortest dependency path connecting the two candidate entities (BACTERIA and HABITAT/GEOGRAPHICAL) in the collapsed Stanford Dependency graph.

We initialize word representations using pretrained vectors created using six billion words of biomedical text (PubMed and PMC documents). During training, the pre-trained word embeddings are fine-tuned while randomly initialized POS and dependency type representations are trained from scratch. We showed that as the number of training examples is very limited, the random initialization of the network can considerably impact the quality of the learned model. To address this issue, we introduced a voting approach that aggregates the outputs of differently initialized neural network models. Different aggregation thresholds can be used to select different precision-recall trade-offs. Using this method, we showed that our proposed deep neural network can be efficiently trained to have good generalization for unseen data even with minimal training data. Our method ranked second among the entries to the shared task, achieving an F-score of $52.1 \%$ with $62.3 \%$ precision and $44.8 \%$ recall.

There are a number of open questions regarding our model that we hope to address in future work. First, we observed how the initial random state of the model can impact its final performance on unseen data. It is interesting to investigate whether (and to what extent) pre-training the POS and dependency type embeddings can address this issue. One possible approach would be to apply the method to similar biomedical relation extraction tasks that include larger corpora than the BB3-event task (Pyysalo et al., 2008) and use the learned POS and dependency embeddings for ini- tialization for this task. This could also establish to what extent pre-training these representations can boost the F-score.

Second, it will be interesting to study how the method performs with different amounts of training data. On one hand, we can examine to what extent the training corpus size can be reduced without compromising the ability of the proposed network to learn the classification task; on the other, we can explore how this deep learning method compares with previously proposed stateof-the-art biomedical relation extraction methods on larger relation extraction corpora.

Third, the method and task represent an opportunity to study how the word embeddings used for initialization impact relation extraction performance and in this way assess the benefits of different methods for creating word embeddings in an extrinsic task with real-world applications.

Finally, it is interesting to investigate different methods to deal with cross-sentence relations. Here we ignored all potential relations where the entities are mentioned in different sentences as there is no path connecting tokens across sentences in the dependency graph. One simple method that could be considered is to create an artificial "paragraph" node connected to all sentence roots to create such paths (cf. e.g. Melli et al. (2007)).

We aim to address these open questions and further extensions of our model in future work.

\section{Acknowledgments}

We would like to thank Kai Hakala, University of Turku, for his technical suggestions for the pipeline development and the anonymous reviewers for their insightful comments and suggestions. We would also like to thank the CSC IT Center for Science Ltd. for computational resources. This work has been supported in part by Medical Research Council grant MR/M013049/1.

\section{References}

Antti Airola, Sampo Pyysalo, Jari Björne, Tapio Pahikkala, Filip Ginter, and Tapio Salakoski. 2008. All-paths graph kernel for protein-protein interaction extraction with evaluation of cross-corpus learning. BMC bioinformatics, 9(11):1.

Sophia Ananiadou, Sampo Pyysalo, Junichi Tsujii, and Douglas B Kell. 2010. Event extraction for sys- 
tems biology by text mining the literature. Trends in biotechnology, 28(7):381-390.

Nguyen Bach and Sameer Badaskar. 2007. A review of relation extraction. Language Technologies Institute, Carnegie Mellon University.

Frédéric Bastien, Pascal Lamblin, Razvan Pascanu, James Bergstra, Ian J. Goodfellow, Arnaud Bergeron, Nicolas Bouchard, and Yoshua Bengio. 2012. Theano: new features and speed improvements. In Proc. Deep Learning and Unsupervised Feature Learning NIPS 2012 Workshop.

Yoshua Bengio, Patrice Simard, and Paolo Frasconi. 1994. Learning long-term dependencies with gradient descent is difficult. IEEE Transactions on Neural Networks, 5(2):157-166.

Jari Björne and Tapio Salakoski. 2013. TEES 2.1: Automated annotation scheme learning in the bionlp 2013 shared task. In Proc. BioNLP Shared Task, pages 16-25.

Jari Björne, Filip Ginter, and Tapio Salakoski. 2012. University of Turku in the BioNLP'11 shared task. BMC Bioinformatics, 13(S-11):S4.

Robert Bossy, Julien Jourde, Philippe Bessières, Maarten van de Guchte, and Claire Nédellec. 2011. Bionlp shared task 2011: Bacteria biotope. In Proc. BioNLP Shared Task, pages 56-64.

Razvan C. Bunescu and Raymond J. Mooney. 2005. A shortest path dependency kernel for relation extraction. In Proc. HLT-EMNLP, pages 724-731.

Razvan Bunescu and Raymond J. Mooney. 2006. Subsequence kernels for relation extraction. In Proc. NIPS.

Eugene Charniak and Mark Johnson. 2005. Coarseto-fine N-best parsing and maxent discriminative reranking. In Proc. ACL, pages 173-180.

Franois Chollet. 2015. Keras. https://github. com/fchollet/keras.

Faisal Mahbub Chowdhury, Alberto Lavelli, and Alessandro Moschitti. 2011. A study on dependency tree kernels for automatic extraction of protein-protein interaction. In Proc. BioNLP 2011, pages 124-133.

Marie-Catherine de Marneffe and Christopher D. Manning. 2008. The stanford typed dependencies representation. In Proc. CrossParser, pages 1-8.

Marie-Catherine de Marneffe, Bill MacCartney, and Christopher D. Manning. 2006. Generating typed dependency parses from phrase structure parses. In Proc. LREC-2006, pages 449-454.

Sepp Hochreiter and Jürgen Schmidhuber. 1997. Long short-term memory. Neural Comput., 9(8):17351780 .
Jin-Dong Kim, Tomoko Ohta, Sampo Pyysalo, Yoshinobu Kano, and Jun'ichi Tsujii. 2009. Overview of BioNLP'09 shared task on event extraction. In Proc. BioNLP Shared Task, pages 1-9.

Jin-Dong Kim, Tomoko Ohta, Sampo Pyysalo, Yoshinobu Kano, and Junichi Tsujii. 2011. Extracting bio-molecular events from literature - the BioNLP'09 shared task. Computational Intelligence, 27(4):513-540.

Diederik P. Kingma and Jimmy Ba. 2014. Adam: A method for stochastic optimization. CoRR, abs/1412.6980.

David McClosky. 2010. Any Domain Parsing: Automatic Domain Adaptation for Natural Language Parsing. Ph.D. thesis.

Gabor Melli, Martin Ester, and Anoop Sarkar. 2007. Recognition of multi-sentence n-ary subcellular localization mentions in biomedical abstracts. In Proceedings of LBM.

Tomas Mikolov, Ilya Sutskever, Kai Chen, Greg S Corrado, and Jeff Dean. 2013. Distributed representations of words and phrases and their compositionality. In Advances in Neural Information Processing Systems 26, pages 3111-3119.

Claire Nédellec, Robert Bossy, Jin-Dong Kim, JungJae Kim, Tomoko Ohta, Sampo Pyysalo, and Pierre Zweigenbaum. 2013. Overview of BioNLP shared task 2013. In Proc. BioNLP Shared Task, pages 1-7.

Truc-Vien T Nguyen, Alessandro Moschitti, and Giuseppe Riccardi. 2009. Convolution kernels on constituent, dependency and sequential structures for relation extraction. In Proc. EMNLP, pages 1378-1387.

Sampo Pyysalo, Antti Airola, Juho Heimonen, Jari Björne, Filip Ginter, and Tapio Salakoski. 2008. Comparative analysis of five protein-protein interaction corpora. BMC bioinformatics, 9(3):1.

Sampo Pyysalo, Filip Ginter, Hans Moen, Tapio Salakoski, and Sophia Ananiadou. 2013. Distributional semantic resources for biomedical text mining. In Proc. LBM, pages 39-44.

Zorana Ratkovic, Wiktoria Golik, Pierre Warnier, Philippe Veber, and Claire Nédellec. 2011. BioNLP 2011 task Bacteria Biotope: The Alvis system. In Proc. BioNLP Shared Task, pages 102-111.

Nitish Srivastava, Geoffrey Hinton, Alex Krizhevsky, Ilya Sutskever, and Ruslan Salakhutdinov. 2014. Dropout: A simple way to prevent neural networks from overfitting. Journal of Machine Learning Research, 15:1929-1958.

Yan Xu, Lili Mou, Ge Li, Yunchuan Chen, Hao Peng, and Zhi Jin. 2015. Classifying relations via long short term memory networks along shortest dependency paths. In Proc. EMNLP, pages 1785-1794. 